\title{
THE AD393 GUEST STAR AND THE SNR RX J1713.7-3946
}

\author{
Z. R. WANG, Q. Y. QU \& Y. CHEN
}

Dept. of Astronomy, Nanjing Univ., Nanjing 210093, PR China

RX J1713.7-3946 is a new and bright supernova remnant (SNR) in soft X-rays discovered by Pfeffermann \& Aschenbach (1996, hereafter PA 1996). Its visual position as shown on its name is within the asterism Wei where the AD393 guest star occured (Wang et al. 1997).

The age of RX J1713.7-3946 was estimated about two thousand years old (PA 1996). It is nearly consistent with the explosion time of the AD393 guest star, 1.6 thousand years ago. The ASCA observation of RX J1713.73946 also supports it to be a young SNR (Koyama et al. 1997).

Since the morphology of RX J1713.7-3946 is central-ring-brightened shell-like in soft X-rays, we have reason to preferably consider it to be a remnant of SNIb/Ic (Chen et al. 1995). The peak luminosity of SNIb/Ic is much lower than SNIa (Nomoto et al. 1995). According to the estimated distance of $1.1 \mathrm{kpc}$ for RX J1713.7-3946 (PA 1996), the visual magnitude of its corresponding SN is consistent with that of the AD393 guest star described in the ancient record (Wang et al. 1997). Therefore, the SNR RX J1713.7-3946 might be the remnant of the AD393 guest star.

Dr. Qu, Q. Y. gratefully acknowledges the support of K. C. Wong Education Foundation, Hong Kong.

\section{References}

Chen, Y., Liu, N., Wang, Z. R. 1995, ApJ, 446,755

Koyama, K., Kinugasa, K., Matsuzaki, K. et al. 1997, PASJ, 49, L7

Nomoto, N., Iwamoto, K., Suzuki, T. 1995, Phys. Rep., 256, 173

Pfeffermann, E., Aschenbach, B. 1996, in Roentgenstrahlung from the Universe, International Conference on X-ray Astronomy \& Astrophysics, MPE Report 263 (eds. H. Zimmermann, J. Trumper \& H. Yorke), p.267

Wang, Z. R., Qu, Q. Y., Chen, Y. 1997, A\&A, 318, L59 